Published in final edited form as:

Cancer Causes Control. 2009 July ; 20(5): 765-773. doi:10.1007/s10552-008-9291-x.

\title{
Prospective study of physical activity and the risk of ovarian cancer
}

\section{Michael F. Leitzmann,}

Nutritional Epidemiology Branch, Division of Cancer Epidemiology and Genetics, National Cancer Institute, National Institutes of Health, Department of Health and Human Services, 6120

Executive Blvd, Rockville, MD 20892, USA

\section{Corinna Koebnick,}

Nutritional Epidemiology Branch, Division of Cancer Epidemiology and Genetics, National Cancer Institute, National Institutes of Health, Department of Health and Human Services, 6120

Executive Blvd, Rockville, MD 20892, USA

Research \& Evaluation, Southern California Permanente Medical Group, Oakland, CA, USA

\section{Steven C. Moore,}

Nutritional Epidemiology Branch, Division of Cancer Epidemiology and Genetics, National Cancer Institute, National Institutes of Health, Department of Health and Human Services, 6120

Executive Blvd, Rockville, MD 20892, USA

Kim N. Danforth,

Hormonal and Reproductive Epidemiology Branch, Division of Cancer Epidemiology and Genetics, National Cancer Institute, National Institutes of Health, Department of Health and Human Services, Rockville, MD, USA

\section{Louise A. Brinton,}

Hormonal and Reproductive Epidemiology Branch, Division of Cancer Epidemiology and Genetics, National Cancer Institute, National Institutes of Health, Department of Health and Human Services, Rockville, MD, USA

\section{Albert R. Hollenbeck, AARP, Washington, DC, USA}

Arthur Schatzkin, and Nutritional Epidemiology Branch, Division of Cancer Epidemiology and Genetics, National Cancer Institute, National Institutes of Health, Department of Health and Human Services, 6120 Executive Blvd, Rockville, MD 20892, USA

\section{James V. Lacey}

Hormonal and Reproductive Epidemiology Branch, Division of Cancer Epidemiology and Genetics, National Cancer Institute, National Institutes of Health, Department of Health and Human Services, Rockville, MD, USA

Michael F. Leitzmann: michael.leitzmann@klinik.uni-regensburg.de

\section{Abstract}

\footnotetext{
(C) Springer Science+Business Media B.V. 2008

Correspondence to: Michael F. Leitzmann, michael . leitzmann@klinik . uni-regensburg. de.

Present Address: M. F. Leitzmann, Institute of Epidemiology and Preventive Medicine, University of Regensburg, Franz-Josef-

Struass-Allee 11, 93053 Regensburg, Germany
} 
Background-Available studies on physical activity and ovarian cancer have produced inconsistent findings, with some previous studies reporting a positive association between vigorous physical activity and ovarian cancer risk.

Methods-We prospectively investigated the relations of self-reported moderate and vigorous physical activity to ovarian cancer in a cohort of 96,216 US women aged 51-72 years at baseline, followed from 1996-1997 to 31 December 2003.

Results-During seven years of follow-up, we documented 309 cases of epithelial ovarian carcinoma. In analyses adjusted for age, the relative risks (RRs) of ovarian cancer for individual and joint combinations of moderate and vigorous physical activity such as entirely inactive, neither moderate nor vigorous physical activity, moderate physical activity only, vigorous physical activity only, and both moderate and vigorous physical activity were $0.88,1.0$ (reference), 0.89, 1.05 , and 1.08 (95\% confidence interval $(\mathrm{CI})=0.81-1.43$, respectively. After multivariate adjustment, the relation was essentially unchanged (RR comparing women with both moderate and vigorous physical activity to those with neither moderate nor vigorous physical activity = $1.10 ; 95 \% \mathrm{CI}=0.82-1.48$ ). The null association between physical activity and ovarian cancer persisted in subgroups of women as defined by body mass index, parity, oral contraceptive use, menopausal hormone therapy, family history of ovarian cancer, and other variables (all $p$ values for interaction $>0.05$ ).

Conclusions-Neither moderate nor vigorous physical activity showed a statistically significant association with ovarian cancer in this large cohort of women.

\section{Keywords}

Physical activity; Cancer; Prospective study

\section{Introduction}

Ovarian cancer is the fourth most common cancer among women in the United States (US) and Europe, with more than 190,000 new cases of ovarian cancer diagnosed worldwide each year [1]. In part, because ovarian cancer may be asymptomatic until advanced stages, patients tend to be diagnosed late in the course of the disease [2] and the five-year survival rate is below $40 \%$ [3]. This underscores the need for better understanding and prevention of ovarian cancer.

It has been hypothesized that physical activity may protect against the development of ovarian cancer. Possible biological mechanisms include reduced body fat and related decreases in estrogen levels, lower ovulation frequency, and diminished chronic inflammation $[4,5]$. Despite plausible etiologic pathways linking increased physical activity to decreased ovarian cancer risk, available epidemiologic data are mixed. Most [6-12], but not all case-control studies [13-15] observed a statistically significant inverse association between physical activity and ovarian cancer. Similarly, four prospective studies [16-19] are consistent with a reduction in ovarian cancer risk related to high versus low levels of physical activity, although four other prospective studies [20-23] did not support an association.

In contrast, three reports [24-26] from two large cohorts found an increased risk of ovarian cancer with greater levels of physical activity. In those cohorts, the positive relation of physical activity to ovarian cancer risk appeared particularly strong for vigorous activity, with approximately two-fold increases in risk of ovarian cancer with high versus low amounts of vigorous activity. Thus, in a large cohort of US women we sought to help clarify 
the relation of physical activity to ovarian cancer and to evaluate the possibility of enhanced risk of ovarian cancer with vigorous physical activity.

\section{Methods}

\section{Study population}

In 1995-1996, 566,407 AARP members (formerly known as the American Association of Retired Persons) aged 50-71 years and residing in one of six US states (CA, FL, LA, NJ, $\mathrm{NC}$, and PA) or two metropolitan areas (Atlanta, GA and Detroit, MI) completed and returned a mailed questionnaire on medical history, diet, and physical activity to initiate the NIH-AARP Diet and Health Study [27]. A second questionnaire that included more detailed information on family history of cancer and menopausal hormone therapy was mailed to baseline questionnaire respondents within six months and was returned by $59.5 \%$ of participants. The study was approved by the Special Studies Institutional Review Board (IRB) of the US National Cancer Institute.

The current analysis included 138,057 potentially eligible women who returned the second questionnaire. We excluded women with previously diagnosed cancer other than nonmelanoma skin cancer before baseline ( $n=9,171$ women, including 1,572 cases of ovarian cancer), those with bilateral oophorectomy before baseline $(n=27,908)$, women with unknown oophorectomy status at baseline $(n=2,164)$, and subjects with missing information on physical activity $(n=2,598)$. After these exclusions, the analytic cohort included 96,216 women.

\section{Cohort follow-up}

Study participants were followed by regular matching of the cohort database to the National Change of Address database maintained by the US Postal Service and through processing of undeliverable mail, other address update services, and directly from participants. Vital status was ascertained by the linkage of the cohort to the Social Security Administration Death Master File. Follow-up searches of presumed deaths in the National Death Index (NDI) Plus provided verification and information on cause of death. For matching purposes, we have virtually complete data on first and last name, address history, gender, and date of birth. Social security number is available for $85 \%$ of our cohort.

\section{Endpoint ascertainment}

Incident cases of epithelial ovarian cancer were identified by probabilistic linkage to the state cancer registries serving our cohort. We recently expanded our cancer registry ascertainment area by three states TX, AZ, and NV to capture cancer cases occurring among participants who moved to those states during follow-up. The North American Association of Central Cancer Registries (NAACCR) certifies all 11 cancer registries [28]. We conducted a validation study comparing registry findings to self-reports and medical records, and found that approximately $90 \%$ of all cancer cases in our cohort were validly identified using linkage to cancer registries [29].

Cancers were identified by anatomic site and histologic code using the International Classification of Disease for Oncology (ICD-O, second and third editions) [30]. The endpoint considered was epithelial ovarian cancer (ICD-O C56.9) with histologic codes for serous carcinoma $(8441,8460,8461)$, mucinous carcinoma $(8470,8471,8480,8481)$, endometroid carcinoma $(8380,8381,8560,8570)$, clear cell carcinoma $(8310,8313)$, and other adenocarcinomas $(8010,8020,8021,8050,8070,8120,8140,8255,8260,8323,8440$, $8450,8562)$. The majority of cases was serous carcinomas ( 159 cases), followed by other adenocarcinomas (102 cases), endometroid carcinomas ( 23 cases), mucinous carcinomas (14 
cases), and clear cell carcinomas (11 cases). Because there were insufficient number of cases of mucinous, endometroid, and clear cell carcinomas, we evaluated associations according to major histologic subtype (serous, non-serous). We also considered ovarian cancer cases according to SEER (Surveillance Epidemiology and End Reports) stage at the initial diagnosis or treatment of the reportable tumor (68 non-metastatic cases, 195 metastatic cases). SEER stage was available for all but 46 cases. In addition, we examined the relation of physical activity to fatal ovarian cancer (231 fatal cases).

\section{Assessment of physical activity}

The baseline questionnaire inquired about structured vigorous exercise during the previous year, defined as the frequency each week spent at activities that lasted $20 \mathrm{~min}$ or more and caused either increases in breathing or heart rate or working up a sweat. There were six possible response options: never; rarely; 1-3 times per month; 1-2 times per week; 3-4 times per week; and 5 or more times per week. The second questionnaire requested information on the average time spent each week at moderate and vigorous activities using the categories of never; rarely; weekly, but less than $1 \mathrm{~h}$ per week; $1-3 \mathrm{~h}$ per week; $4-7 \mathrm{~h}$ per week; and more than $7 \mathrm{~h}$ per week. Specific examples included brisk walking/fast dancing, walking during golf, hiking/mountain climbing, cheerleading/drill team, tennis, biking, swimming, aerobics, jogging/running, rowing, basketball/baseball, football/soccer, handball/racquetball, weight lifting, heavy gardening, and heavy housework. Our physical activity assessment is similar to the Physical Activity Scale for the Elderly (PASE), which showed an intra-class correlation coefficient of 0.84 for two administrations of the questionnaire mailed three to seven weeks apart and a correlation coefficient of 0.58 comparing activity energy expenditure as assessed by the questionnaire with that using doubly labeled water [31].

We combined the information from both the baseline and the second questionnaires to divide participants into five categories according to their physical activity level: (1) entirely inactive (women who reported never or rarely engaging in moderate or vigorous physical activity); (2) neither moderate nor vigorous physical activity (less than $3 \mathrm{~h}$ of moderate activity per week and less than three times $20+$ minutes of vigorous activity per week); (3) moderate physical activity only (more than $3 \mathrm{~h}$ of moderate activity per week and less than three times 20+ minutes of vigorous activity per week); (4) vigorous physical activity only (less than $3 \mathrm{~h}$ of moderate activity per week and three or more times $20+$ minutes of vigorous activity per week); and (5) both moderate and vigorous physical activity (more than $3 \mathrm{~h}$ of moderate activity per week and three or more times $20+$ minutes of vigorous activity per week). The group of subjects who fell into the category of neither moderate nor vigorous physical activity served as the reference group.

\section{Statistical analysis}

Each participant accrued follow-up time beginning at the scan date of the second questionnaire and ending at the date of diagnosis of epithelial ovarian carcinoma, move out of the registry ascertainment area, death, or the end of follow-up on 31 December 2003, whichever occurred first. We used Cox proportional hazards regression [32] to estimate hazard ratios and $95 \%$ confidence intervals (CI) while controlling for multiple variables simultaneously. We tested for and found no departures from the proportional hazards assumption by age, calendar period, or duration of follow-up. We assessed epithelial ovarian carcinoma risk in two models, one model adjusting for age, and a second model additionally adjusting for body mass index (BMI), height, race/ethnicity; smoking status, family history of ovarian cancer, parity, use of oral contraceptives, age at menarche, age at menopause, and menopausal hormone therapy use. 
To examine whether the association between physical activity and risk of ovarian cancer was modified by other factors, we conducted tests for multiplicative interaction by modeling physical activity, the variable of interest, and the products of physical activity and the variable of interest (the interaction terms); the statistical significance of the latter was evaluated using a likelihood-ratio test. We also evaluated the relation of physical activity to epithelial ovarian carcinoma within strata of those variables. All relative risks (RRs) are presented with $95 \% \mathrm{CI}$, and the reported $p$ values are based on two-sided tests. All analyses were conducted using SAS release 8.2 (SAS Institute, Cary, NC).

\section{Results}

Among the 96,216 women included in the analysis, $30 \%$ were classified as engaging in both moderate and vigorous physical activity, whereas $29 \%$ did not participate in either moderate or vigorous physical activity and $7 \%$ were entirely physically inactive; $20 \%$ fell into the category of moderate physical activity only; $14 \%$ fell into the category of vigorous physical activity only.

Participants who engaged in both moderate and vigorous activity were more likely to use oral contraceptives, menopausal hormone therapy, and to be former or never smokers compared to those who did not participate in either moderate or vigorous activity (Table 1). Physically more active women also had a lower BMI than physically less active women.

During 649,599 person-years of follow-up, we documented 309 cases of epithelial ovarian cancer. In age-adjusted analysis, increasing levels of physical activity (entirely inactive; neither moderate nor vigorous physical activity; moderate physical activity only; vigorous physical activity only; and both moderate and vigorous physical activity) were not associated with ovarian cancer risk (Table 2). The RR comparing women with both moderate and vigorous activity to those with neither moderate nor vigorous activity was $1.08(95 \% \mathrm{CI}=0.81-1.43)$. The multivariate-adjusted risk estimate was very similar $(\mathrm{RR}=$ $1.10 ; 95 \% \mathrm{CI}=0.82-1.48)$.

To evaluate whether the association between physical activity and ovarian cancer was modified by menopausal status, we repeated our analysis after limiting the cohort to postmenopausal women ( $n=6$ ovarian cancer cases excluded). Results were materially unaltered (multivariate RR for both moderate and vigorous activity vs. neither moderate nor vigorous activity $=1.15 ; 95 \% \mathrm{CI}=0.86-1.55$ ).

Because undiagnosed ovarian cancer may have caused lower physical activity levels at the time the questionnaires were administered, we repeated our analysis after excluding all cases of ovarian cancer diagnosed during the first two years of follow-up ( $n=95$ ovarian cancer cases excluded). Findings were similar to overall (multivariate RR comparing women with both moderate and vigorous activity to those with neither moderate nor vigorous activity = $1.12 ; 95 \% \mathrm{CI}=0.79-1.59$ ). Risk estimates were also virtually unchanged when we further minimized any impact that undiagnosed ovarian cancer may have had on physical activity levels by additionally excluding subjects who reported poor health at entry ( $n=99$ ovarian cancer cases excluded; corresponding $\mathrm{RR}=1.11 ; 95 \% \mathrm{CI}=0.78-1.58$ ).

We evaluated relations according to the major histologic subtype of ovarian cancer (Table 2). The multivariate RR of serous ovarian cancer comparing women with both moderate and vigorous activity to those with neither moderate nor vigorous activity was $1.42(95 \% \mathrm{CI}=$ $0.93-2.17)$. That relation was virtually entirely contributed by vigorous activity. The multivariate RR of serous ovarian cancer comparing women with vigorous activity to those with neither moderate nor vigorous activity was $1.46(95 \% \mathrm{CI}=0.89-2.40)$. In contrast, a weaker relation with serous ovarian carcinoma was found with moderate activity. The 
multivariate RR of serous ovarian cancer comparing women with moderate activity to those with neither moderate nor vigorous activity was $1.16(95 \% \mathrm{CI}=0.71-1.89)$. For the combined group of endometroid, mucinous, clear cell, and other adenocarcinomas, the multivariate-adjusted relative risk comparing women with both moderate and vigorous activity to those with neither moderate nor vigorous activity was 0.87 (95\% CI $=0.57-1.32$ ).

We also examined associations according to tumor stage (Table 2). We found a pattern of decreasing risk of non-metastatic ovarian cancer and increasing risk of metastatic ovarian cancer across increasing intensity levels of physical activity. However, the point estimates relating vigorous activity to non-metastatic and metastatic ovarian cancer were similar. The multivariate RR of non-metastatic ovarian cancer comparing women with vigorous activity to those with neither moderate nor vigorous activity was $1.11(95 \% \mathrm{CI}=0.53-2.32)$. A similar relation was found between vigorous activity and metastatic ovarian carcinoma (RR $=1.06 ; 95 \% \mathrm{CI}=0.67-1.68$ ). The multivariate $\mathrm{RR}$ of fatal ovarian cancer comparing women with vigorous activity to those with neither moderate nor vigorous activity was 1.13 $(95 \% \mathrm{CI}=0.75-1.72)$.

We investigated whether the relation of physical activity to ovarian cancer was altered by potential effect modifiers (Table 3 ). The null association between physical activity and ovarian cancer persisted in subgroups of women as defined by BMI, parity, oral contraceptive use, menopausal hormone therapy, family history of ovarian cancer, and other variables (all $p$ values for interaction $>0.05$ ).

\section{Discussion}

In this prospective study of middle-aged to elderly US women, we found no inverse relation between physical activity and ovarian cancer. In fact, our data do not entirely exclude the possibility of a positive relation between a high amount of vigorous activity and serous ovarian carcinoma, although results were statistically non-significant. We detected no statistically significant relation between moderate and vigorous physical activity and ovarian cancer in subgroups of women defined by BMI, parity, oral contraceptive use, menopausal hormone therapy, family history of ovarian cancer, non-steroidal anti-inflammatory drug (NSAID) use, and other variables.

A recent meta-analysis [8] on the relation of physical activity to ovarian cancer pooled the results from available cohort studies and reported an inverse relation that was statistically non-significant $(\mathrm{RR}=0.81 ; 95 \% \mathrm{CI}=0.57-1.17$; high vs. low physical activity) and showed evidence for heterogeneity between individual studies $(p=0.004)$. That heterogeneity was driven by data from two cohort studies $[24,25]$ suggesting that increased recreational physical activity, particularly vigorous activity, was associated with a greater risk of ovarian cancer. Specifically, the Iowa Women's Health Study [24] observed that, compared with physically inactive women, those engaging in vigorous physical activity five or more times per week had a RR of 2.52 (95\% CI = 1.01-6.28). An updated analysis in that cohort [26] including more than twice the number of cases reported a corresponding relative risk of 2.38 (95\% CI $=1.29-4.38$ ). Similarly, the Nurses' Health Study [25] found RRs of 1.58 (95\% CI $=1.05-2.38)$ and $1.48(95 \% \mathrm{CI}=0.89-2.48)$ for women engaging in vigorous recreational activity three to four times per week and five or more times per week, respectively, as compared with women not engaging in vigorous recreational activity.

Among the remaining studies evaluated in the meta-analysis [8], one cohort study [16] reported a strong decrease in ovarian cancer risk associated with increased physical activity $(\mathrm{RR}=0.33 ; 95 \% \mathrm{CI}=0.16-0.67)$, whereas three other prospective studies [17-19] were 
consistent with an approximate 30\% reduction in ovarian risk related to high versus low levels of physical activity, but the findings were not statistically significant.

Four cohort studies [20-23] did not support an association between physical activity and ovarian cancer. These non-supportive cohort studies [20-23] were all conducted in Europe or China, and in three of those studies [20,21,23] the exposure was defined as occupational physical activity assessed by job codes. Specifically, one Finnish cohort study [20] found no difference in ovarian cancer risk among physical education teachers (assumed to have higher lifetime physical activity levels) than language teachers (assumed to have a lower lifetime physical activity levels). One retrospective cohort study from Denmark [21] presented a standardized incidence ratio for ovarian cancer of $0.94(95 \% \mathrm{CI}=0.19-2.75)$ for low versus high level of occupational activity (based on $n=3$ ovarian cancer cases). A study from China [23] reported standardized incidence ratios of 84 for housewives and 132 for technical workers. We did not examine occupational activity because only a very small number of women reported physically demanding jobs in our cohort, rendering an analysis of occupational activity in our study uninformative. We also reasoned that occupational activity is unlikely to be a meaningful population-level source of physical activity among comparable groups of AARP-eligible women.

Results from case-control studies of physical activity and ovarian cancer have been somewhat more but not entirely consistent than those from prospective studies. Specifically, six case-control studies [5, 7, 9-12] observed a statistically significant 20-50\% decreased risk of ovarian cancer for high versus low physical activity, whereas two case-control studies $[13,14]$ are compatible with a $30 \%$ reduction in risk but the results were not statistically significant, and two case-control studies $[8,15]$ reported a null association. The overall pooled data from available case-control studies on physical activity and ovarian cancer [8] generated a statistically significant inverse association $(\mathrm{RR}=0.79 ; 95 \% \mathrm{CI}=$ $0.70-0.81)$.

The finding of a suggestively positive association between vigorous activity and serous ovarian cancer in our study raises concern of a potential biological basis. Alternatively, the positive relation between physical activity and ovarian cancer could be attributable to detection bias if more vigorously active women tended to be more symptomatic. Although there were limited numbers of ovarian cancer cases according to tumor stage in our dataset, we evaluated the possibility of detection bias by examining relations according to tumor stage. Indeed, we found a pattern suggestive of increasing risk of metastatic ovarian cancer across increasing intensity levels of physical activity, whereas a pattern indicative of decreasing risk of non-metastatic ovarian cancer across increasing intensity levels of physical activity was observed. In addition, no obvious pattern was noted between physical activity and fatal ovarian cancer. These exploratory analyses support the possibility of detection bias. The potential for a non-causal positive relation of physical activity to ovarian cancer requires further investigation in this and other future studies.

In theory, physical activity may reduce ovarian cancer risk through numerous biological mechanisms, including reduced adiposity leading to decreased levels of estrogen or growth factors, lower ovulation frequency, and reduced chronic inflammation [33]. Such factors may also modify the physical activity and ovarian cancer relation. The Nurses' Health Study suggested that the positive relation of physical activity to ovarian cancer was more pronounced among lean women [25]. The Breast Cancer Detection Demonstration Project (BCDDP) reported a more pronounced inverse relation of physical activity to ovarian cancer among parous than nulliparous women [18]. However, those interactions were not confirmed in the current or other previous studies [17, 19, 22]. Likewise, we found no effect modification of the physical activity and ovarian cancer relation by NSAID use. 
The main limitation of the current study is the potential measurement error in our assessment of physical activity [34]. We lacked data to quantify the degree of misclassification of physical activity levels among women in our study, but measurement error would have needed to be substantial to obscure a large or moderate-sized association. However, we cannot rule out the possibility of a small physical activity effect. The large size of our cohort with the related costs did not permit us to employ more accurate physical activity instruments, such as activity monitors. An additional potential limitation of our study is that we did not assess potential changes in activity levels during follow-up.

An important strength of the current study is its prospective design in which we assessed physical activity before ovarian cancer diagnosis and excluded from the analyses subjects with pre-existing cancer at baseline. This helped to reduce the influence that malignant disease may have had on recalled or reported physical activity levels at study onset. In secondary analyses, we further minimized the potential for reverse causation due to preexisting but undiagnosed ovarian cancer by excluding the first two years of follow-up and excluding participants with poor health status at entry.

We adjusted for most of the important known ovarian cancer risk factors. Results from the age-adjusted and multivariate models were very similar, suggesting that confounding by risk factors, we were able to measure was minimal. Although the overall number of cases in our study was modest, we had greater statistical power than many previous cohort studies to examine whether relations between physical activity and ovarian cancer differed for population subgroups.

In summary, our analysis does not provide support for an inverse association between physical activity and ovarian cancer. Our results add to very limited available evidence of a positive relation between vigorous activity and ovarian cancer. However, findings were statistically non-significant and could have been due to detection bias as evidenced by an enhanced stage of disease across increasing activity intensity levels but no association with fatal ovarian cancer. Our data generally confirm the accumulating body of evidence from prospective epidemiologic studies showing that physical activity is unlikely to play an important role in the etiology of ovarian cancer.

\section{Acknowledgments}

We are indebted to the participants in the NIH-AARP Diet and Health Study for their outstanding cooperation. We also thank Sigurd Hermansen and Kerry Grace Morrissey from We-stat for study outcomes ascertainment and management, Leslie Carroll at Information Management Services for data support and analysis, and Tawanda Roy at the Nutritional Epidemiology Branch for research assistance.

Grant support This research was supported by the Intramural Research Program of the NIH, National Cancer Institute.

\section{References}

1. Ferlay, J.; Bray, F.; Pisani, P.; Parkin, DM.; Globocan. IARC Cancer Base. Lyon, France: 2000. Cancer incidence, mortality and prevalence worldwide; p. 2004

2. Aletti GD, Gallenberg MM, Cliby WA, Jatoi A, Hartmann LC. Current management strategies for ovarian cancer. Mayo Clin Proc. 2007; 82(6):751-770. [PubMed: 17550756]

3. Jemal A, Siegel R, Ward E, Hao Y, Xu J, Murray T, Thun MJ. Cancer statistics. CA Cancer J Clin. 2008; 58(2):71-96. [PubMed: 18287387]

4. Lukanova A, Kaaks R. Endogenous hormones and ovarian cancer: epidemiology and current hypotheses. Cancer Epidemiol Biomarkers Prev. 2005; 14(1):98-107. [PubMed: 15668482]

5. Ness RB, Cottreau C. Possible role of ovarian epithelial inflammation in ovarian cancer. J Natl Cancer Inst. 1999; 91(17):1459-1467. [PubMed: 10469746] 
6. Cottreau CM, Ness RB, Kriska AM. Physical activity and reduced risk of ovarian cancer. Obstet Gynecol. 2000; 96(4):609-614. [PubMed: 11004368]

7. Freedman DM, Dosemeci M, McGlynn K. Sunlight and mortality from breast, ovarian, colon, prostate, and non-melanoma skin cancer: a composite death certificate based case-control study. Occup Environ Med. 2002; 59(4):257-262. [PubMed: 11934953]

8. Olsen CM, Bain CJ, Jordan SJ, Nagle CM, Green AC, Whiteman DC, Webb PM. Recreational physical activity and epithelial ovarian cancer: a case-control study, systematic review, and metaanalysis. Cancer Epidemiol Biomarkers Prev. 2007; 16(11):2321-2330. [PubMed: 18006921]

9. Pan SY, Ugnat AM, Mao Y. Physical activity and the risk of ovarian cancer: a case-control study in Canada. Int J Cancer. 2005; 117(2):300-307. [PubMed: 15898119]

10. Riman T, Dickman PW, Nilsson S, Nordlinder H, Magnusson CM, Persson IR. Some life-style factors and the risk of invasive epithelial ovarian cancer in Swedish women. Eur J Epidemiol. 2004; 19(11):1011-1019. [PubMed: 15648594]

11. Tavani A, Gallus S, La VC, Dal ML, Negri E, Pelucchi C, Montella M, Conti E, Carbone A, Franceschi S. Physical activity and risk of ovarian cancer: an Italian case-control study. Int J Cancer. 2001; 91(3):407-411. [PubMed: 11169967]

12. Zhang M, Lee AH, Binns CW. Physical activity and epithelial ovarian cancer risk: a case-control study in China. Int J Cancer. 2003; 105(6):838-843. [PubMed: 12767071]

13. Bertone ER, Newcomb PA, Willett WC, Stampfer MJ, Egan KM. Recreational physical activity and ovarian cancer in a population-based case-control study. Int J Cancer. 2002; 99(3):431-436. [PubMed: 11992414]

14. Chiaffarino F, Parazzini F, Bosetti C, Franceschi S, Talamini R, Canzonieri V, Montella M, Ramazzotti V, Franceschi S, La VC. Risk factors for ovarian cancer histotypes. Eur J Cancer. 2007; 43(7):1208-1213. [PubMed: 17376671]

15. Dosemeci M, Hayes RB, Vetter R, Hoover RN, Tucker M, Engin K, Unsal M, Blair A. Occupational physical activity, socioeconomic status, and risks of 15 cancer sites in Turkey. Cancer Causes Control. 1993; 4(4):313-321. [PubMed: 8347780]

16. Schnohr P, Gronbaek M, Petersen L, Hein HO, Sorensen TI. Physical activity in leisure-time and risk of cancer: 14-year follow-up of 28,000 Danish men and women. Scand J Public Health. 2005; 33(4):244-249. [PubMed: 16087486]

17. Biesma RG, Schouten LJ, Dirx MJ, Goldbohm RA, van den Brandt PA. Physical activity and risk of ovarian cancer: results from the Netherlands Cohort Study (The Netherlands). Cancer Causes Control. 2006; 17(1):109-115. [PubMed: 16411060]

18. Hannan LM, Leitzmann MF, Lacey JV Jr, Colbert LH, Albanes D, Schatzkin A, Schairer C. Physical activity and risk of ovarian cancer: a prospective cohort study in the United States. Cancer Epidemiol Biomarkers Prev. 2004; 13(5):765-770. [PubMed: 15159308]

19. Patel AV, Rodriguez C, Pavluck AL, Thun MJ, Calle EE. Recreational physical activity and sedentary behavior in relation to ovarian cancer risk in a large cohort of US women. Am J Epidemiol. 2006; 163(8):709-716. [PubMed: 16495470]

20. Pukkala E, Poskiparta M, Apter D, Vihko V. Life-long physical activity and cancer risk among Finnish female teachers. Eur J Cancer Prev. 1993; 2(5):369-376. [PubMed: 8401170]

21. Soll-Johanning H, Bach E. Occupational exposure to air pollution and cancer risk among Danish urban mail carriers. Int Arch Occup Environ Health. 2004; 77(5):351-356. [PubMed: 15108001]

22. Weiderpass E, Margolis KL, Sandin S, Braaten T, Kumle M, Adami HO, Lund E. Prospective study of physical activity in different periods of life and the risk of ovarian cancer. Int J Cancer. 2006; 118(12):3153-3160. [PubMed: 16425259]

23. Zheng W, Shu XO, McLaughlin JK, Chow WH, Gao YT, Blot WJ. Occupational physical activity and the incidence of cancer of the breast, corpus uteri, and ovary in Shanghai. Cancer. 1993; 71(11):3620-3624. [PubMed: 8490910]

24. Mink PJ, Folsom AR, Sellers TA, Kushi LH. Physical activity, waist-to-hip ratio, and other risk factors for ovarian cancer: a follow-up study of older women. Epidemiology. 1996; 7(1):38-45. [PubMed: 8664399] 
25. Bertone ER, Willett WC, Rosner BA, Hunter DJ, Fuchs CS, Speizer FE, Colditz GA, Hankinson SE. Prospective study of recreational physical activity and ovarian cancer. J Natl Cancer Inst. 2001; 93(12):942-948. [PubMed: 11416116]

26. Anderson JP, Ross JA, Folsom AR. Anthropometric variables, physical activity, and incidence of ovarian cancer: the Iowa Women's Health Study. Cancer. 2004; 100(7):1515-1521. [PubMed: 15042687]

27. Schatzkin A, Subar AF, Thompson FE, Harlan LC, Tangrea J, Hollenbeck AR, Hurwitz PE, Coyle L, Schussler N, Michaud DS, Freedman LS, Brown CC, et al. Design and serendipity in establishing a large cohort with wide dietary intake distributions : the National Institutes of HealthAmerican Association of Retired Persons Diet and Health Study. Am J Epidemiol. 2001; 154(12): 1119-1125. [PubMed: 11744517]

28. >NAACR. Standards for completeness, quality, analysis, and management of data. Springfield, IL: North American Association of Central Disease Registries; 2004.

29. Michaud DS, Midthune D, Hermansen S, Leitzmann MF, Harlan LC, Kipnis V, Schatzkin A. Comparison of cancer registry case ascertainment with SEER estimates and self-reporting in a subset of the NIH-AARP Diet and Health Study. J Regist Manag. 2005; 32(2):70-75.

30. Fritz, AG.; Percy, C.; Jack, A.; Shanmugaratnam, K.; Sobin, LH.; Parkin, DM.; Whelan, S. 3rd edn.. Geneva, Switzerland: World Health Organization (WHO); 2000. International classification of diseases for oncology: ICD-O.

31. Marshall AL, Smith BJ, Bauman AE, Kaur S. Reliability and validity of a brief physical activity assessment for use by family doctors. Br J Sports Med. 2005; 39(5):294-297. [PubMed: 15849294]

32. Cox DR. Regression models and life tables. J R Stat Soc (B). 1972; 34:187-220.

33. McTiernan A. Mechanisms linking physical activity with cancer. Nat Rev Cancer. 2008; 8(3):205211. [PubMed: 18235448]

34. Sallis JF, Saelens BE. Assessment of physical activity by self-report: status, limitations, and future directions. Res Q Exerc Sport. 2000; 71(2 Suppl):S1-S14. [PubMed: 10925819] 


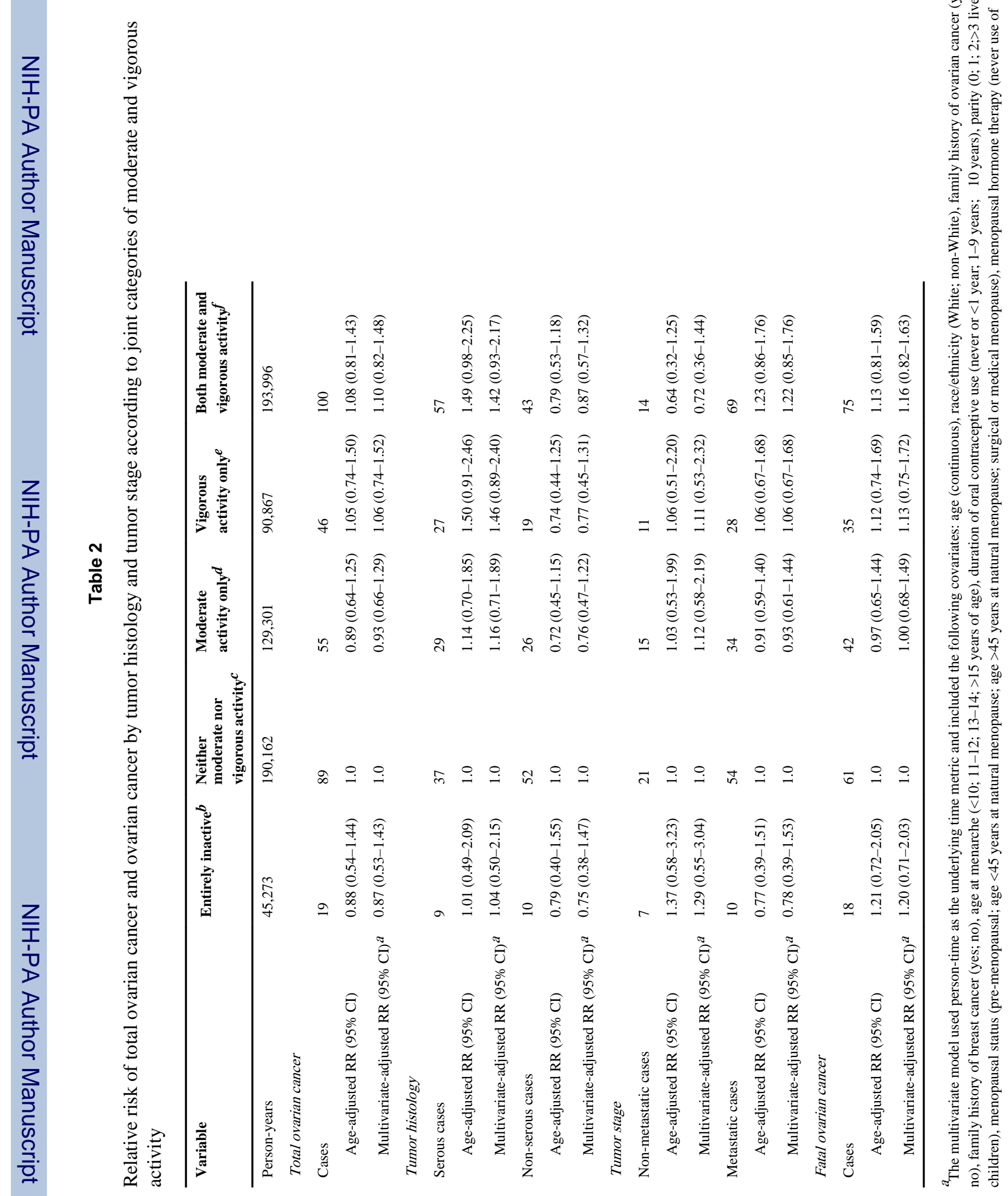




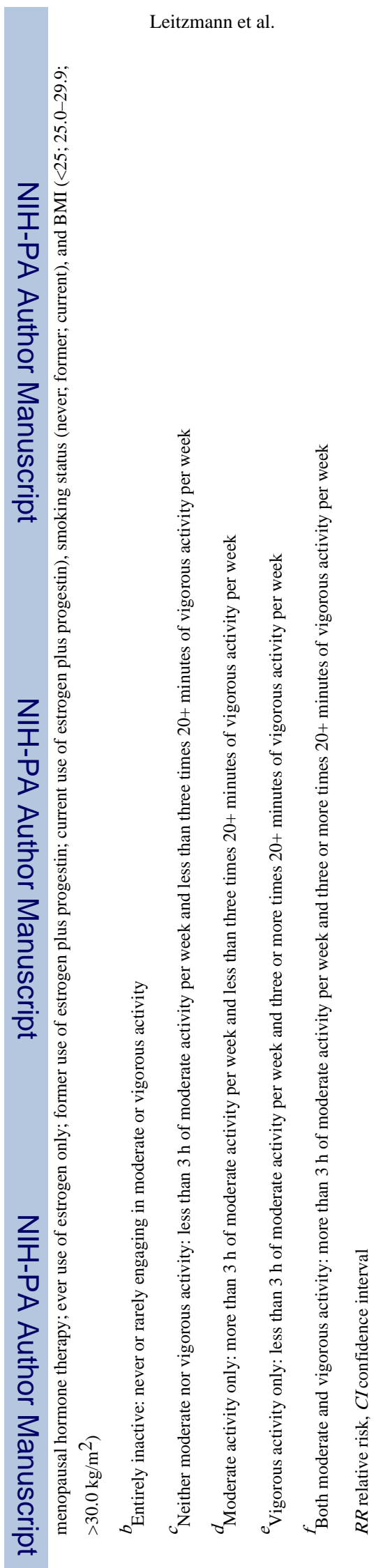




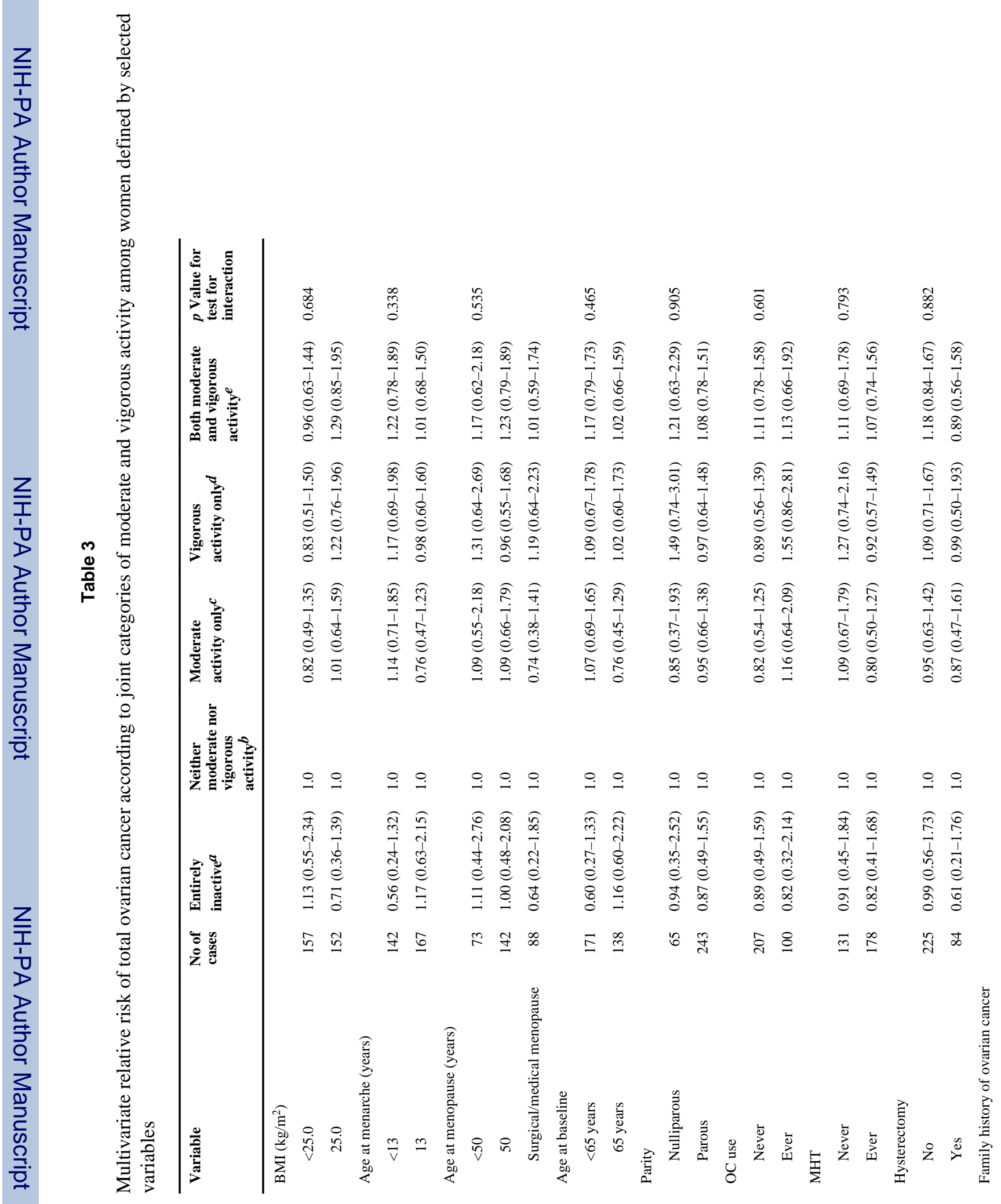




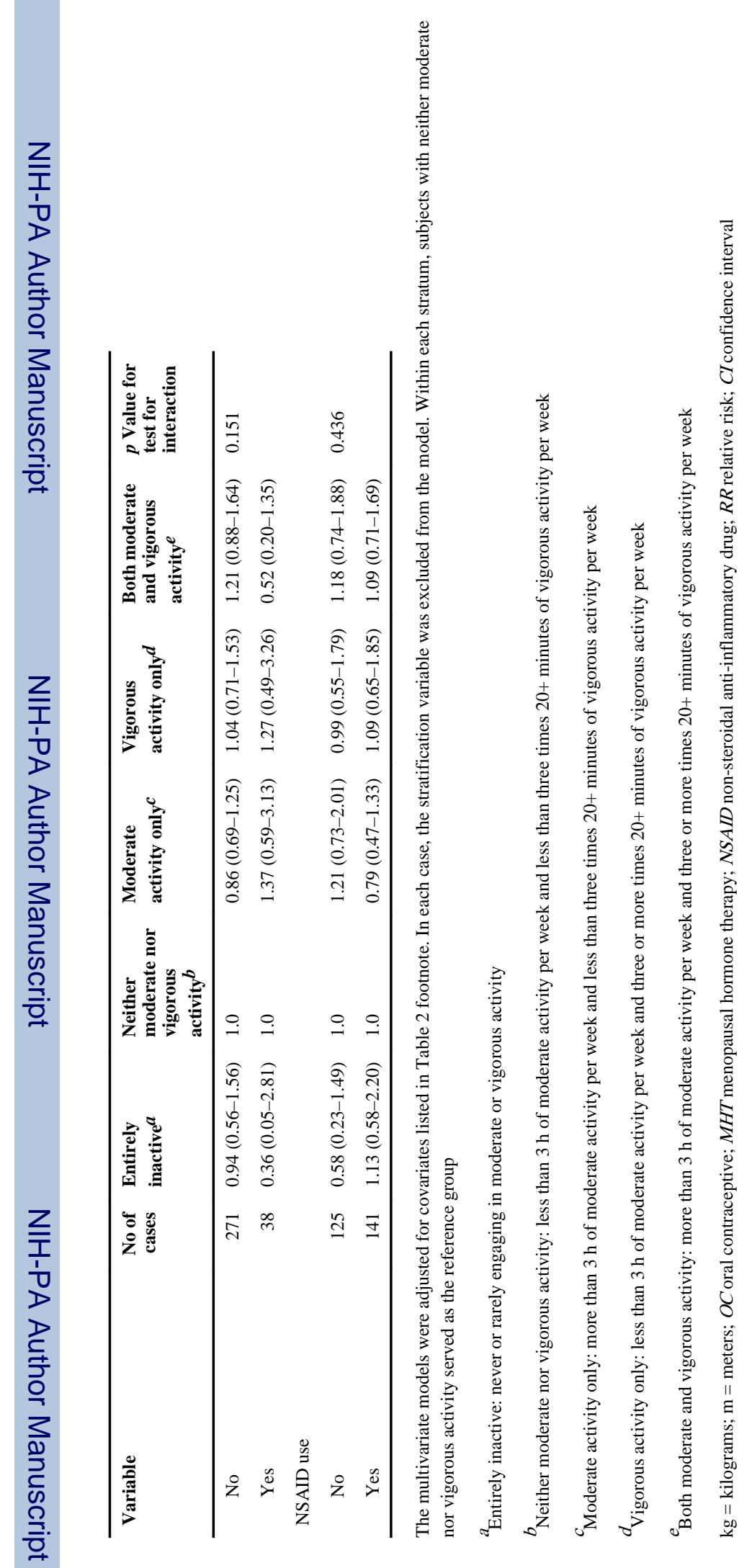

